5. García-Zamalloa A, Ruiz-Irastorza G, Aguayo FJ, Gurrutxaga N. Pseudochylothorax. Report of 2 cases and review of the literature. Medicine (Baltimore) 1999; 78: 200-7.

6. Nogueras C, Monteagudo M, Vila M, Cabezuelo A, Mariscal D, Berlanga E. Recent-onset tuberculous pleurisy presenting as pseudochylothorax. Am J Med 2002; 113: 166-8.

\section{Ascitis y edemas en extremidades inferiores}

\section{Sr. Director:}

La pericarditis constrictiva puede clasificarse en la forma típica (crónica o subaguda), efusivo-constrictiva, localizada y oculta. Una historia de varios años de duración y el predominio de la ascitis en los hallazgos clínicos, simulando una enfermedad hepática, es típica. Se produce una reducción en el llenado diastólico de todas las cavidades del corazón y determina su volumen diastólico. Así, casi todo el llenado ventricular se produce al inicio de la diástole, hasta que el volumen intracardíaco alcanza el límite establecido por el pericardio no distensible (1). En la exploración, el dato más relevante es la elevación de la presión venosa yugular, con un marcado seno Y. Puede existir un incremento inspiratorio paradójico en la presión venosa central (signo de Kussmaul) (2). El diagnóstico diferencial con la miocardiopatía restrictiva sigue siendo dificultoso, pero actualmente se cuentan con distintas exploraciones que en conjunto deben hacernos llegar a un diagnóstico (ECG, cateterización cardiaca, TAC, RMN, etc.) (3).

Paciente varón de 86 años que ingresa en Medicina Interna por presentar edemas progresivos en extremidades inferiores, llegando hasta genitales, con aumento del perímetro abdominal de unos dos meses de evolución, y disnea de moderados esfuerzos con disminución de la diuresis en los últimos quince días. No presentaba ortopnea, ni disnea paroxística nocturna. Entre sus antecedentes personales destacan: úlcus duodenal, diagnosticado en 1998 por endoscopia, con clínica de pirosis, y un episodio de flutter auricular 4:1 con insuficiencia cardiaca cinco meses antes del ingreso. No hábitos tóxicos.

En la exploración destacaban importantes edemas en extremidades inferiores, edema de pared abdominal, más evidente en zonas declives, semiología de ascitis moderada e ingurgitación yugular muy evidente incluso en sedestación y signo de Kussmaul. Tensión arterial de 95/60 mmHg. No existencia de pulso paradójico.

En los exámenes complementarios realizados en Urgencias el hemograma fue normal, en la bioquímica se apreció urea y creatinina elevadas $(1,09 \mathrm{~g} / \mathrm{L}$ y $2 \mathrm{mg} / \mathrm{dL}$, respectivamente) con sodio normal y potasio de $5,3 \mathrm{mEq} / \mathrm{L}$, en la radiografía de tórax había leve cardiomegalia, sin signos de descompensación cardíaca y en el electrocardiograma se apreció un ritmo sinusal a 66 1.p.m., llamando la atención los bajos voltajes de forma difusa.

El paciente recibió tratamiento con dieta pobre en sal, restricción hídrica y furosemida endovenosa, y posteriormente vía oral. Las diuresis fueron abundantes, pasando de un peso de $81,5 \mathrm{~kg}$ a $76 \mathrm{~kg}$ en diez días. En el ecocardiograma-doppler se apreciaron variaciones de las curvas de flujo de llenado y expulsión ventricular con la respiración, septo con movimiento anómalo sensible también a las variaciones respiratorias, con aurícula derecha dilatada pero no los ventrículos, con una función sistólica de ventrículo izquierdo conservada e impresión de

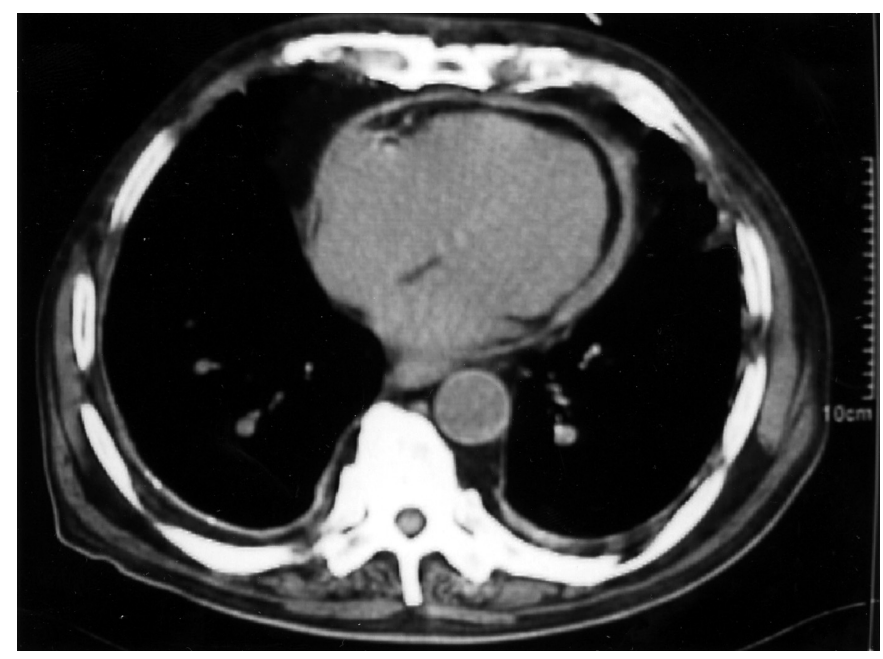

Fig. 1. TAC toraco-abdominal: adenopatías pre y pararaqueales.

pericardio engrosado. En la TAC toraco-abdominal (Fig. 1) se apreciaron adenopatías pre y para traqueales, pericardio con 4 $\mathrm{mm}$ de grosor, ausencia de dilatación de cavidades derechas, densidad miocárdica homogénea, engrosamiento y calcificaciones pleurales, dilatación moderada de las venas cavas y ascitis cuantiosa. La prueba de la tuberculina y las baciloscopias en esputo fueron negativas. Los edemas y la ascitis disminuyeron, permaneciendo presente la ingurgitación yugular y el signo de Kussmaul, dando de alta el paciente con furosemida vía oral, con el diagnóstico de pericarditis constrictiva.

En un $42 \%$, de los casos de pericarditis constrictiva, la etiología es desconocida. La tuberculosis, la cirugía o radiación mediastínica, además de enfermedades autoinmunes sistémicas y otras como las neoplasias pueden ser la causa. El tratamiento de la misma es la pericardiectomía, pues sólo una minoría de pacientes sobreviven muchos años con dieta y diuréticos (4). La mortalidad operatoria está entre el 5 y el $15 \%$, y tiene un pronóstico global a largo plazo influido por la clase funcional preoperatoria, la presencia de insuficiencia renal,... afectando de forma desfavorable.

Así, es posible que no deba intentarse la pericardiectomía de manera sistemática en pacientes muy ancianos o en aquellos con una esperanza de vida limitada, dado el riesgo elevado quirúrgico y el pronóstico posterior, como es el caso de nuestro paciente.

\section{Toyas Miazza, M. R. Ortas Nadal' ${ }^{1}$ B. Ordóñez Rubio, S. González Azuara, P. S. del Río Martínez}

Servicio de Medicina Interna B y ${ }^{I}$ Cardiología. Hospital Clínico Universitario Lozano Blesa. Zaragoza

1. Beverly H. Lorell. Enfermedades pericárdicas. En: Tratado de Cardiología Braunwald. McGraw-Hill Interamericana editores. Pericarditis constrictiva. Mexico: Healthcare Group 1999; 1635-44.

2. Bilchick KC, Wise RA. Paradoxical physical findings described by Kussmaul: pulsus paradoxical and KussmaulÅLs sign. Lancet 2002; 359: 1940-42.

3. Hancock EW. Differential diagnosis of restrictive cardiomyopathy and constrictive pericarditis. Heart 2001; 86: 343-9.

4. Hoit BD. Management of effusive and constrictive pericardial heart disease. Circulation 2002; 105: 2939-42. 